\title{
Does Enhanced Primary Care enhance primary care? Policy-induced dilemmas for allied health professionals
}

\author{
Michele M Foster, Geoffrey Mitchell, Terry Haines, Sean Tweedy, Petrea Cornwell and Jennifer Fleming
}

A

third of the problems encountered in primary care general practice are now chronic in nature. ${ }^{1}$ The Enhanced Pri-

mary Care (EPC) program, introduced in 1999, is one of a range of initiatives aimed at improving prevention and management of chronic disease in Australia. EPC is consistent with Wagner's chronic care model in that the patient's personal physician remains the locus of care, but care quality is enhanced through integrated multiprofessional input. ${ }^{2}$ Other initiatives that aim to improve management of chronic disease include diseasespecific initiatives (eg, diabetes), and incentives for practice organisations (eg, the Practice Incentives Program [PIP]) to attain certain public health targets. Since its introduction, EPC has been modified in response to areas of perceived need and to address potential barriers to general practitioner participation arising from complex administrative processes.

One modification is introduction of the Chronic Disease Management (CDM) Medicare items, which allow Medicare rebates to be paid for individual allied health professional (AHP) services. This policy was introduced in 2004 under Strengthening Medicare. Rebates are paid if the patient is eligible (ie, they have a chronic or complex health condition) and AHP services are delivered under an EPC care plan and coordinated by a GP (Box 1). Up to five allied health visits are available per patient per year. ${ }^{3}$ The CDM items are designed to provide more coordinated quality care and better access to multidisciplinary team care. This represents a positive step towards delivery system change.

However, a critical question is whether the current policy can deliver effective and equitable care to people who most need it. We question whether the current reimbursement system supports comprehensive and effective management of chronic conditions by AHPs. With caps on annual visits, care may be compromised by discretionary management (by both medical and allied health practitioners) of the limitations imposed by policy. ${ }^{4}$ This includes discretion about which AHPs should be involved and how the allocated sessions are used. Given the fee-for-service model of primary care, we also question whether the current policy strategy will advance equity of access to allied health services. Is the "onesize-fits-all" approach appropriate?

\section{Will subsidised allied health services facilitate comprehensive management of chronic conditions?}

Effective chronic care relies on delivery systems that support continuous and quality interactions with the care team, tailored care according to need, and cooperation among care providers. ${ }^{5}$ Support by non-medical providers for self-management and behaviour change, and continuous follow-up to assess clinical improvement and self-management competence are also important. ${ }^{6}$ The restriction to five sessions per year suggests an emphasis on assessment and brief intervention rather than the sophisticated mix of care advocated for effective chronic care. Team Care Arrangements (TCAs) require that the GP and at least two other health professionals must take part in the care of the patient. ${ }^{3}$ If both of these other professionals are private AHPs, they have to
ABSTRACT

- One aim of Medicare's Enhanced Primary Care (EPC) initiative is to encourage multidisciplinary care of patients with chronic disease by funding five allied health treatment sessions per patient per year.

- In many cases, the number of funded treatments is far less than standard clinical practice indicates, particularly when the five visits are shared between service providers.

- We believe clinical outcomes may be compromised by adhering to the funded hours, and inequity of outcome may arise based on socioeconomic status and the ability of patients to pay.

- Research that determines how patients and allied health practitioners are responding to this initiative is required.

- Research is also required to evaluate whether EPC enhances clinical outcomes compared with no allied health intervention and standard allied health practice.

MJA 2008; 188: 29-32

\section{Flow diagram of individual allied health services under Medicare Chronic Disease Management items}

General practitioner

Allied health professionals (AHPs)

Preparation of GP Management

Plan (GPMP)

Item 721 Medicare fee $\$ 124.95$

and

Coordination Team Care

Arrangements (TCAs)

Item 723 Medicare fee $\$ 98.95$

GP collaborating with participating

providers on required treatment

services

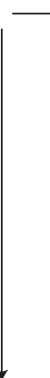

Referral to eligible AHPs — may involve initial consultation about TCA

Not rebated

Provision of individual AHP services maximum five sessions per patient year may be shared between two AHPs

Medicare fee $\$ 53.90$ Rebate $\$ 45.85$

Review of GPMP

Item 725 Medicare fee $\$ 62.50$

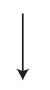

Written report to GP on first and last visit, if providing multiple services, otherwise after each service Not rebated

Coordination of a Review of TCA Item 727 Medicare fee $\$ 62.50$

Recommended once every 6 months or earlier if clinically required

May involve AHPs collaborating with GPs

Not rebated 
share the five sessions. The GP determines how the sessions are apportioned.

Although evidence about the specific frequency of allied health services associated with improved outcomes for different chronic conditions is limited, five subsidised sessions may not be adequate to facilitate improvement for some people, and will, we believe, often prevent AHPs providing care in line with recommended clinical guidelines (see Box 2 for an example). According to guidelines for stroke, people living in the community more than 6 months after stroke should have access to interventions to improve fitness and mobility, as well as be provided with information and a range of support to self-manage. ${ }^{7}$ Under the CDM items, AHPs may be forced to develop treatment strategies that are at variance with current recommended practice. Further, although five sessions may be adequate for mild conditions, the lack of flexibility fails to acknowledge variations in treatment requirements for different conditions or for people with coexisting chronic conditions (Box 3).

The PIP is designed to provide more flexibility by allowing general practices to employ nurses and AHPs. These incentives have generally failed to outweigh the financial and organisational barriers facing practices. ${ }^{8}$ New allied health items from May 2007 , allowing rebates for group services provided to patients with type 2 diabetes, also provide more flexibility. However, this is more likely to be appropriate for patients with less complex needs. Patients with other diseases, such as musculoskeletal conditions, are also likely to benefit from such interventions, but are currently outside the Medicare funding umbrella.

A likely disincentive to AHP participation in chronic care management is the lack of remuneration for other activities integral to multidisciplinary team care (Box 1). Unlike GPs and private physicians, AHPs are not remunerated for participating in case conferences for planning or review purposes. ${ }^{9}$ The development of a TCA requires communication between the GP and other team members. Ideally, AHPs should see the patient before contributing to a care plan. Neither an assessment consultation nor phone contact with the GP is remunerated. Essentially, this policy forces AHPs to engage in pro-bono work, which is at odds with operating a financially viable small business. The policy reinforces the notion of allied health services being "optional" rather than central to multidisciplinary team care. This may discourage AHPs from participating in more than a minimalist way in multidisciplinary activities, and is likely to reduce the quality and effectiveness of chronic care.

Thus, although the contribution of AHPs to primary care management of chronic conditions is being recognised, their role is being significantly prescribed. AHPs are being encouraged to contribute to care management and to participate in multidisciplinary team care, but under conditions that do not take maximum advantage of their clinical expertise in tailoring care plans for individuals and facilitating the self-management and behaviour change required. How AHPs elect to account for these constraints when providing services will be critical in determining the success of the current policy.

\section{Will Medicare-subsidised allied health services facilitate equity of access?}

The likelihood of copayments and existing pressures on the public health system may be problematic if wanting to ensure equity of access to allied health care. Under the CDM items, the Medicare

\section{Case example: exercise physiologist services for moderate osteoarthritis}

Aim: to facilitate a self-managed, home-based exercise program for patient with moderate osteoarthritis

\section{Standard clinical practice}

Time required is 6-9 hours, comprising:

- Pre- and post-intervention measures (1.5-2 hours) of physical (eg, pain, strength, functioning) and behavioural (eg, stage of change and exercise self-efficacy) assessment;

- Graduated, individualised introduction to evidence-based exercise in combination with stage-matched behaviour change strategies (eg, enhancement of client rapport, self-efficacy, problem solving) (4-7 hours); and

- Provider-initiated inter-professional contact (0.5-1 hour, unbilled).

\section{Enhanced Primary Care}

Total time available under Team Care Arrangement is five sessions. Scenario assumes three exercise physiology and two other allied health sessions (eg, physiotherapy, podiatry). Exercise physiology would comprise:

- Abbreviated pre- and post-intervention measures - emphasise physical measures (1 hour); and

- Abbreviated intervention delivery (2 hours):

$>$ less individualisation of exercise and tailoring of behaviour change strategies (ie, greater use of simplified exercise templates and general exercise adherence strategies);

$>$ decreased time for behaviour change strategies; and

$>$ no scope for additional sessions where client has adverse response or is behaviourally unresponsive.

Note: a Team Care Arrangement may require 1-2.5 hours of unpaid interprofessional interaction by the practitioner if Case Conferencing (Item 740744) or Team Care Arrangement Reviews (Item 727) are required.

rebate can either be accepted by the AHP as the complete payment (bulk-billing), or be supplemented with a copayment. Cost may be a barrier for some patients if bulk-billing is not offered, given that services provided by most AHPs generally cost more than the scheduled fee. ${ }^{10}$ Copayments are a deterrent to access to necessary care, and to timely care, particularly for lower socioeconomic groups. ${ }^{11}$ Moreover, people who forego care because of cost often suffer a higher occurrence of chronic disease than those who seek care. ${ }^{12}$ If services are not offered under bulk-billing, or are not readily available through the public sector, then access to allied health services will likely be inequitable. ${ }^{13}$ People who can afford private health insurance are better placed to avoid waiting lists and limitations on resources. ${ }^{11}$

How do practitioners respond to the limited support offered by Medicare? Three options may arise.

- Practitioners may recommend that patients pay for additional allied health services to receive more comprehensive care. Although this is consistent with encouraging more individual responsibility for health care, it raises the potential of inequity, with differential treatment patterns based on socioeconomic status and the ability to support private health insurance coverage or copayments. Patients may choose not to pay for allied health services above the subsidised services, thereby receiving limited services and possibly subtherapeutic care. This may increase the societal burden of health care provision without improving health outcomes and averting future health care costs. 


\section{Case examples: patients with increasing complexity of type 2 diabetes and use of allied health services subsidised under Medicare}

\section{GP Management Plan and allied health group services}

Mary, 55 years old, was diagnosed with type 2 diabetes secondary to obesity 6 months ago. She lives with (and is primary carer for) an elderly parent, and works full-time in the public service. She has tried to follow her general practitioner's advice to lose weight and increase her exercise, but reports that this has been difficult. A full assessment by the GP revealed she has lost $5 \mathrm{~kg}$ over 6 months (body mass index [BMI], $38 \mathrm{~kg} / \mathrm{m}^{2}$ ), but her blood glucose level remains high. The GP discusses the possibility of Mary attending a group program to learn more about managing diabetes, and she is keen to attend.

The GP prepares a GP Management Plan and collaborates with a diabetes educator to include Mary in a group program following assessment for suitability to attend group allied health services.

GP Management Plan review, Team Care Arrangement, and chronic disease management allied health services: adequate services John, 60 years old, was diagnosed with type 2 diabetes 8 months ago, and has a long history of obesity. He lives with his wife and is semi-retired.

At his GP Management Plan review, a full assessment revealed that John has maintained his weight $\left(B M I, 42 \mathrm{~kg} / \mathrm{m}^{2}\right)$ despite repeated information provided regarding the need for weight loss, his blood glucose level is elevated, moderate hypertension is evident, and he reports that a wound on his toe has been slow to heal.

The GP again discusses the need to make changes to his life, such as losing weight and increasing exercise, and the complications that may develop secondary to diabetes. John seems resistant to selfmanaging his diabetes, but would be willing to attend sessions with allied health professionals to have them assist him with managing his diabetes.

The GP completes a Team Care Arrangement after consulting a dietitian to review, manage and monitor John's weight and nutritional status, and a podiatrist to complete a foot examination and educate John about good foot care.

GP Management Plan review, Team Care Arrangement, and chronic disease management allied health services: insufficient services

Bill, 72 years old, has a long-standing diagnosis of type 2 diabetes, had a right lower leg amputation 6 years ago, and has a recent diagnosis of emphysema. He lives alone and is retired.

A full assessment revealed that Bill's blood glucose level is slightly elevated $(6.8 \mathrm{mmol} / \mathrm{L})$, his weight is stable, and he has recently developed an ulcer on his left foot. Discussions with Bill reveal he is no longer attending the hospital clinic for reviews because of transportation difficulties, and he is decreasing participation in activities because of general weakness and shortness of breath. He is having difficulties with activities of daily living (ADLs) (personal and around the house).

The GP decides a Team Care Arrangement would be appropriate, involving a podiatrist to complete routine foot examinations, an occupational therapist to complete assessment of ADLs and prescribe home modifications or equipment, a dietitian to review and monitor Bill's diet, and an exercise physiologist to increase tolerance to exercise.

The GP has to prioritise services and, after collaborating with a number of allied health professionals, refers Bill to an occupational therapist ( 2 sessions), exercise physiologist ( 2 sessions) and podiatrist ( 1 session), although all professionals indicated that this was a less than optimal number of sessions.
- AHPs may shorten assessments and modify clinical practice to complete an abbreviated version of the intervention within the allotted sessions if it is apparent at the outset that the patient is unable or unwilling to pay for additional sessions. Whether this will improve health outcomes for these individuals is a critical question. It is possible that marginal or no improvement can be achieved within the allotted number of sessions.

- Practitioners may refer the patient to a Commonwealth or statefunded allied health service after the subsidised sessions. Although this is likely to improve health outcomes for these patients in the long term, it will create inefficiency, as assessments will be repeated and treatment programs reformulated by the new therapists. It also confers further costs and inconvenience for the patient, and unnecessarily defers resolution of the problem. Long waiting times or lack of relevant professionals in some services may add to the disruption.

\section{Researching the way forward}

The Medicare initiatives for allied health services are a positive step towards more effective chronic care. However, it is questionable whether the current funding and reimbursement arrangements create a framework that encourages recommended clinical practice by AHPs. Moreover, although equity of access is a major issue for the health care system, it is questionable whether current administrative arrangements for allied health services advance equitable care for everyone with chronic conditions.

Research on the implementation of this policy must focus on the participation service provision patterns of AHPs, with particular emphasis on the effect of capped service provision on professional practice and patient outcomes. Comparison of the provision of allied health services and clinical outcomes under the capped remuneration system with recommended best practice will provide insight into the appropriateness and adequacy of the current administration and funding systems. Given the potential for socioeconomic status to compromise delivery of quality and equitable care to all who need it, research also needs to consider the effect of the current program on the personal choices exercised by patients in relation to health care.

\section{Competing interests}

None identified.

\section{Author details}

Michele M Foster, PhD, Senior Lecturer, School of Social Work and Applied Human Sciences ${ }^{1}$

Geoffrey Mitchell, FRACGP, PhD, Associate Professor, PHCRED Senior Research Fellow, School of Medicine ${ }^{1}$

Terry Haines, BPhysiother(Hons), GCertHealthEcon, PhD, Conjoint Physiotherapy Research Fellow, ${ }^{1,2}$

Sean Tweedy, BHMS, MHMS, PhD, MAIC Research Fellow Physical Activity and Disability, School of Human Movement Studies ${ }^{1}$

Petrea Cornwell, BSpPath, PhD, Conjoint Speech Pathology Research Fellow ${ }^{1,2}$

Jennifer Fleming, BOccThy(Hons), PhD, Conjoint Occupational Therapy Senior Research Fellow ${ }^{1,2}$

1 University of Queensland, Brisbane, QLD.

2 Princess Alexandra Hospital, Brisbane, QLD.

Correspondence: m.foster@social.uq.edu.au 


\section{HEALTH CARE}

\section{References}

1 Britt $\mathrm{H}$, Miller GC, Knox S, et al. General practice activity in Australia 2004-05. Canberra: AlHW, 2005. (AlHW Cat. No. GEP 18.) http:// www.aihw.gov.au/publications/index.cfm/title/10189 (accessed Oct 2007).

2 Wagner EH. Chronic disease management: what will it take to improve care for chronic illness? Effective Clin Pract 1998: 1: 2-4.

3 Medicare Australia. Medical Benefits Schedule. Canberra: Medicare Australia, 2006. http://www9.health.gov.au/mbs/ (accessed Mar 2007).

4 Fleming J, Foster M, Taylor S. Contemporary health care practice. In: Taylor S, Foster M, Fleming J, editors. Health care practice in Australia: policy, context and innovations. Melbourne: Oxford University Press, 2007.

5 Wagner E, Austin B, Davis C, et al. Improving chronic illness care: translating evidence into action. Health Aff (Millwood) 2001; 20: 64-78.

6 Wagner $\mathrm{E}$. The role of patient care teams in chronic disease management. BMJ 2000; 320: 569-572.

7 National Stroke Foundation. Clinical guidelines for stroke rehabilitation and recovery. Melbourne: National Stroke Foundation, 2005. http:// www.nhmrc.gov.au/publications/synopses/cp105syn.htm (accessed Oct 2007).

8 Harris M, Harris E. Facing the challenges: general practice in 2020. Med J Aust 2006; 185: 122-124.

9 Harris M. Case conferences in general practice: time for a rethink? Med J Aust 2002; 177: 93-94.

10 Williams L. Jostling for position: a sociology of allied health. In: Germov J, editor. Second opinion: an introduction to health sociology. 3rd ed. Melbourne: Oxford University Press, 2005.

11 Schoen C, Davis K, DesRoches C, et al. Health insurance markets and income inequality: findings from an international health policy survey. Health Policy 2000; 51: 67-85.

12 Elofsson S, Undén A-L, Krakau I. Patient charges - a hindrance to financially and psychosocially disadvantage groups seeking care. Soc Sci Med 1998; 46: 1375-1380.

13 Schofield D. Ancillary and specialist health services: the relationship between income, user rates and equity of access. Aust J Soc Issues 1999; 34: 79-96.

(Received 2 Apr 2007, accepted 12 Sep 2007) 